\title{
Animal Venom for Medical Usage in Pharmacopuncture in Korean Medicine: Current Status and Clinical Implication
}

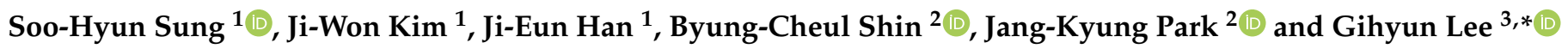 \\ 1 Department of Policy Development, National Development Institute of Korean Medicine, Seoul 04554, Korea; \\ koyote10010@nikom.or.kr (S.-H.S.); cactus@nikom.or.kr (J.-W.K.); jieun2342@nikom.or.kr (J.-E.H.) \\ 2 Division of Clinical Medicine, School of Korean Medicine, Pusan National University, Yangsan 50612, Korea; \\ drshinbc@pusan.ac.kr (B.-C.S.); vivat314@pusan.ac.kr (J.-K.P.) \\ 3 College of Korean Medicine, Dongshin University, Naju 58245, Korea \\ * Correspondence: glee@khu.ac.kr
}

check for

updates

Citation: Sung, S.-H.; Kim, J.-W.;

Han, J.-E.; Shin, B.-C.; Park, J.-K.; Lee,

G. Animal Venom for Medical Usage

in Pharmacopuncture in Korean

Medicine: Current Status and Clinical

Implication. Toxins 2021, 13, 105.

https://doi.org/10.3390/toxins

13020105

Received: 29 December 2020

Accepted: 28 January 2021

Published: 1 February 2021

Publisher's Note: MDPI stays neutral with regard to jurisdictional claims in published maps and institutional affiliations.

Copyright: (C) 2021 by the authors. Licensee MDPI, Basel, Switzerland. This article is an open access article distributed under the terms and conditions of the Creative Commons Attribution (CC BY) license (https:// creativecommons.org/licenses/by/ $4.0 /)$.

\begin{abstract}
Animal venoms, widespread throughout the world, are complex mixtures, the composition of which depends on the venom-producing species. The objective of this study was to contribute to the development of animal venom-based medicines by investigating the use of animal venom pharmacopuncture in Korean medicine (KM) institutions. We surveyed 256 public health centers from 1 through 31 October 2019 as guided by the Ministry of Health and Welfare (MoHW). A questionnaire developed by an expert group was distributed and collected for statistical analysis. The survey identified three types of animal venom-based pharmacopuncture: bee, snake, and toad venoms. The medications are based on a single animal venom ingredient and produced in 11 external herbal dispensaries (EHDs). Each animal venom is processed, refined, and freeze-dried in a cleanroom to produce a powder formulation that is later measured, diluted, filtered, filled, sealed, sterilized, and packaged as pharmacopuncture injections used in KM institutions. Bee venom therapy is effective in treating musculoskeletal pain, snake venom therapy is effective in controlling bleeding during surgery, and toad venom therapy is effective in cancer treatment. The study suggests that bee, snake, and toad venoms could be used in medical institutions and have the potential for drug development.
\end{abstract}

Keywords: animal venom; bee venom; snake venom; toad venom; pharmacopuncture

Key Contribution: Pharmacopuncture of bee, snake, and toad venoms are being used in Korean medicine institutions. Clinical evidence for the efficacy of these animal venoms has been summarized through a literature search. This research contributes to the development of animal venom-based medicines.

\section{Introduction}

Animal venoms, widespread worldwide, are composed of a variety of proteins and peptides that were developed through millions of years of evolution [1-3]. They are complex mixtures that vary depending on the venom-producing species [2]. The most known and studied venoms originate from bees, snakes, scorpions, and spiders [1,2].

Venomous animals are a valuable resource for the development of therapeutics [4]. Bee venom therapy is used to treat musculoskeletal diseases (e.g., low back pain, knee osteoarthritis), Parkinson's disease, adhesive capsulitis, and polycystic ovary syndrome [5]; snake venom-based drugs are used to treat hypertension, heart attack, acute coronary syndrome, stroke, pulmonary embolism, and other diseases [6]. While animal venoms are toxic, they can have therapeutic effects [7].

Korean medicine (KM) institutions have been using animal venoms in their pharmacopuncture therapies: animal venoms are injected into acupunctural points using syringes [8]. Pharmacopuncture, also known as herbal acupuncture, is a new form of acupuncture treatment that injects herbal medicine into acupuncture points [9]. In South 
Korea, pharmacopuncture treatment is one of the most commonly used methods in traditional Korean medicine clinics [10]. Official insurance claims statistics for pharmacopuncture are only available for automobile insurance coverage; it has been reported that approximately 1,555,000 pharmacopuncture sessions were administered to 168,089 patients for related treatments in 2014 [11].

Chinese pharmaceutical companies produce acupunctural medicines approved by the food and drug authorities [12]; these medicinal materials are used in Chinese medicine institutions to treat patients. In South Korea, such medicinal products are made in external herbal dispensaries (EHDs) that employ the level of good manufacturing practice (GMP) required for injections [13]. EHDs are auxiliary facilities of KM institutions that prepare and supply various types of medicines (e.g., capsules, tablets, powders, pharmacopuncture, and decoction) to KM institutions [14].

The purpose of this study was to review the current use of animal venom pharmacopuncture medicines in KM institutions; how such medicines are prepared; for what disease treatment they are being used; and further, to provide basic data for the progressive, knowledge-based development of animal venom treatments derived from the understanding of the current status.

\section{Results}

\subsection{Demographic Characteristics of Pharmacopuncture-EHD (P-EHD)}

A total of 11 Pharmacopuncture-EHD (P-EHD) questions were included in this study. The characteristics of the respondents in this survey are shown in Additional file 1 in Supplementary Materials. The average number of pharmacopuncture medicines produced in the 11 P-EHDs was average 18.4.

\subsection{Preparation Status of Pharmacopuncture}

A total of 1,851,896 vials of 46 different pharmacopuncture medicines were produced in 11 P-EHDs in 2018 alone. The status of the top 20 frequently prepared pharmacopuncture medicines produced in P-EHDs and their ingredients are shown in Table 1.

Table 1. Status and composition of pharmacopuncture.

\begin{tabular}{|c|c|c|c|c|}
\hline NO. & Pharmacopuncture & Composition of Pharmacopuncture & Amount of Preparation (vial) & Ratio of Preparation \\
\hline 1 & Jungseong-eohyeol & $\begin{array}{l}\text { Salviae Radix, Persicae Semen, } \\
\text { Commiphora myrrha Engler, Caesalpinia } \\
\text { sappan Linné, Olibanum, Paeoniae } \\
\text { Radix, Corydalis yanhusuo W.T.Wang, } \\
\text { Gardeniae Fructus }\end{array}$ & $1,373,686$ & $23.45 \%$ \\
\hline 2 & Bee venom & Bee venom & 779,931 & $13.32 \%$ \\
\hline 3 & Hwanglyeon-haedog & $\begin{array}{l}\text { Coptidis Rhizoma, Phellodendri Cortex, } \\
\text { Scutellariae Radix, Gardeniae Fructus }\end{array}$ & 556,064 & $9.49 \%$ \\
\hline 4 & Cheogchusin & $\begin{array}{c}\text { Paeoniae Radix, Osterici seu } \\
\text { Notopterygii Radix et Rhizoma, Araliae } \\
\text { continentalis Radix, Cibotii Rhizoma, } \\
\text { Eucommiae Cortex, Saposhnikoviae } \\
\text { Radix, Acanthopanacis Cortex, } \\
\text { Achyranthis Radix }\end{array}$ & 490,990 & $8.38 \%$ \\
\hline 5 & Haleupagopitumgeun & Harpagophyti Radix & 272,330 & $4.65 \%$ \\
\hline 6 & Jagyag-gamcho & $\begin{array}{c}\text { Paeoniae Radix, Glycyrrhizae Radix et } \\
\text { Rhizoma }\end{array}$ & 153,530 & $2.62 \%$ \\
\hline 7 & Hong-hwa & Carthami Flos, Carthami tinctorii Semen & 146,380 & $2.50 \%$ \\
\hline 8 & Jahageo & Hominis Placenta & 115,909 & $1.98 \%$ \\
\hline 9 & Wild ginseng & Wild ginseng & 48,431 & $0.83 \%$ \\
\hline 10 & Soyeom & $\begin{array}{c}\text { Lonicerae japonicae Flos, Rehmanniae } \\
\text { Radix, Forsythiae Fructus, Gardeniae } \\
\text { Fructus }\end{array}$ & 20,275 & $0.35 \%$ \\
\hline
\end{tabular}


Table 1. Cont.

\begin{tabular}{ccccc}
\hline NO. & Pharmacopuncture & Composition of Pharmacopuncture & Amount of Preparation (vial) & Ratio of Preparation \\
\hline 11 & Jug-yeom & Bamboo salt & 8898 & $0.15 \%$ \\
\hline 12 & Haeng-in & Armeniacae Semen & 7528 & $0.13 \%$ \\
\hline 13 & Snake venom & Snake venom & 5291 & $0.09 \%$ \\
\hline 14 & Cho-o & Aconiti Seoulense Tuber & 4433 & $0.08 \%$ \\
\hline 15 & Yang-geumhwa & Datura metel Linné & 3530 & $0.06 \%$ \\
\hline 16 & Chilpi & Rhus verniciflua Stokes & 3000 & $0.05 \%$ \\
\hline 17 & Cheong-ganbohyeol & Mori Folium & 2600 & $0.04 \%$ \\
\hline 18 & Toad venom & Toad venom & 2114 & $0.04 \%$ \\
\hline 19 & Miso & Salmon milt & 2000 & $0.03 \%$ \\
\hline 20 & Mahwang-cheono & Ephedrae Radix, Aconiti Tuber & 1910 & $0.03 \%$ \\
\hline
\end{tabular}

The following quantities of animal venom preparations were made: bee venom $(99.06 \%, n=779,931)$, snake venom $(0.67 \%, n=5291)$, and toad venom $(0.27 \%, n=2114)$. All of these were composed of a single venom (Figure 1 ).

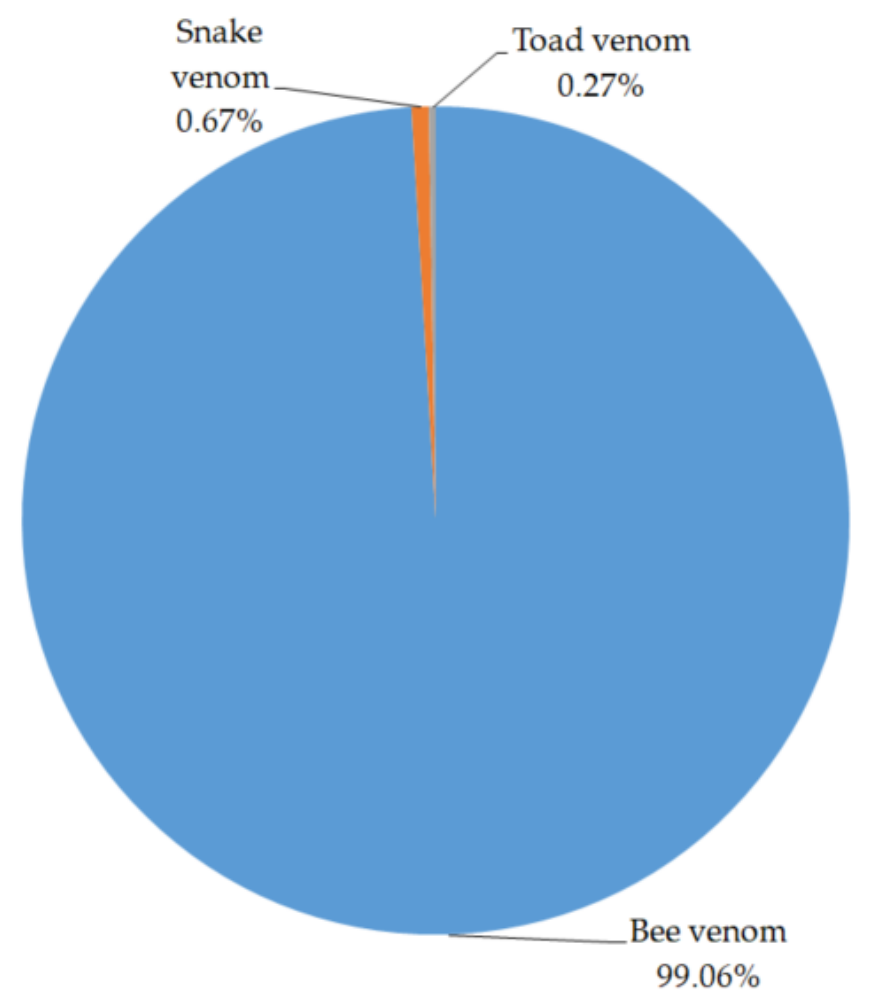

Figure 1. Preparation status of animal venom pharmacopuncture.

\subsection{The Preparation Process of Animal Venom Pharmacopuncture}

Bee venom is extracted from the venom pouch of worker bees (Apis mellifera, ligustica) [15]. Apply a weak current of a low voltage, 3-6V, to a living bee. Then, take the venom coming out of the stinger [15]. Snake venom is extracted from the venom gland of Agkistrodon halys Pallas [15]. Hold a snake by its head and neck. Then, place a vessel under its fangs and press the head where the venom gland is located gently to press the venom out of it. This process is called "snake milking" [15,16]. Toad (Bufo bufo gargarizans Canto) venom is extracted from the skin and parotid glands [15]. The production process of the injectable pharmacopuncture is the same for all three venoms (Figure 2). Venoms are processed, refined, and freeze-dried in a clean room into a powder formulation [15]. The venom powder goes through purification and dilution using distilled water in a specific concentration [15]. The venom is then filled, sealed, sterilized, and packaged as pharmacopuncture injections for clinical use (Figure 2). 


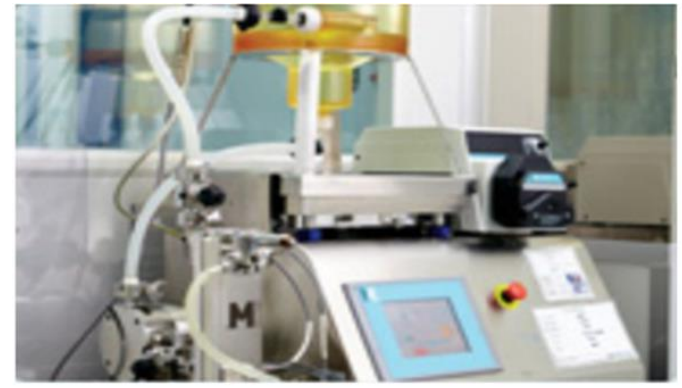

(a)

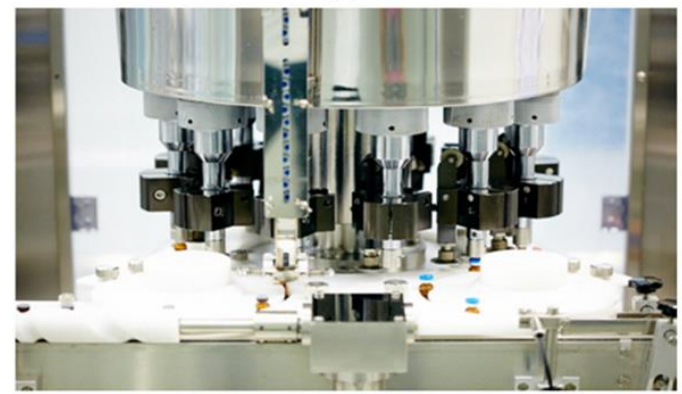

(c)

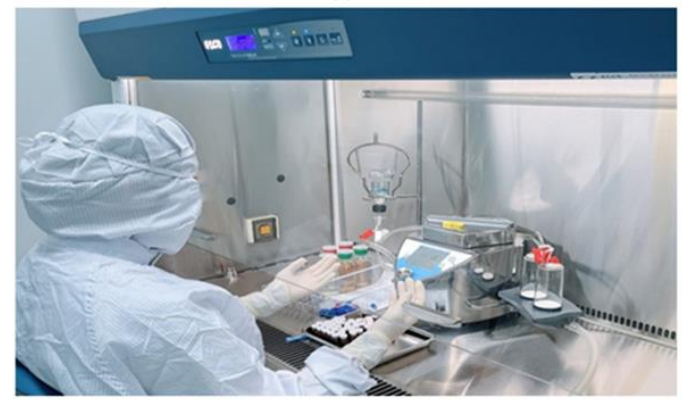

(e)

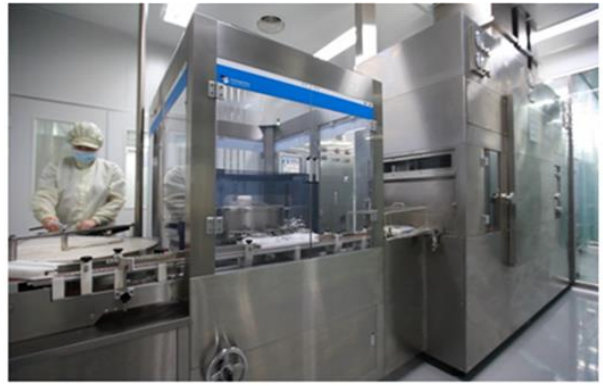

(b)

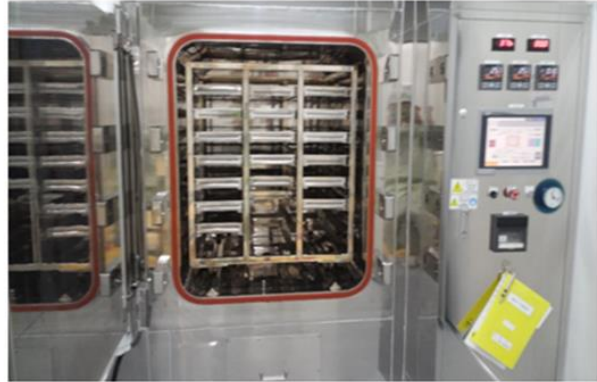

(d)

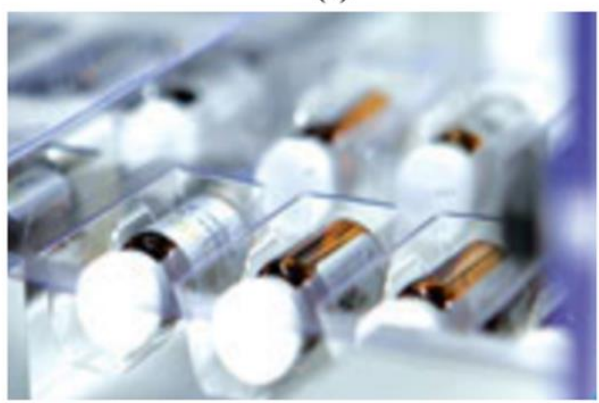

(f)

Figure 2. Preparation process of animal venom pharmacopuncture. Animal venom is prepared with pharmacopuncture through the following process; (a) venom is filted for removing impurities and diluted with distilled water (b) vials are cleaned and sterilized (c) venom is filled and sealed into each vial (d) prepared pharmacopuncture is sterilized (e) quality inspections (e.g., sterility test, endotosin test, and insoluble particulate matter test and $\mathrm{pH}$ test) are conducted and (f) paking pharmacopuncture for clinical use.

\subsection{Clinical Effectiveness of Animal Venom Therapy}

Table 2 summarizes the clinical evidence for animal venom therapy use and efficacy [17-61]. Bee venom therapy is effective in musculoskeletal pain such as post-stroke shoulder pain [18,42-45], shoulder pain [19], chronic low back pain [23-25], neck pain [32], temporomandibular disorder [34], wrist sprain [35], and acute ankle sprain [37,38]. Snake venom therapy has been reported to be clinically effective in hemorrhage control in bi-maxillary orthognathic surgery [50], fracture-related hip hemiarthroplasty [51], and transvesical prostatic adenomectomy [54]. The clinical indications of toad venom therapy include gastric cancer [57], liver cancer [58], and metastatic bone tumors [59]. Eighteen studies $[17-20,22-25,31,32,37,40,41,43,48,57-59]$ reported moderate to severe adverse events in the animal venom treatment groups. 
Table 2. Clinical evidence of animal venom therapy.

\begin{tabular}{|c|c|c|c|c|c|}
\hline $\begin{array}{l}\text { Type of Animal } \\
\text { Venom }\end{array}$ & $\begin{array}{l}\text { Treatment } \\
\text { Diseases }\end{array}$ & $\begin{array}{c}\text { Intervention } \\
\text { (Concentration, Treatment Sessions, } \\
\text { Amount of Venom Use) }\end{array}$ & $\begin{array}{l}\text { Clinical } \\
\text { Effects }\end{array}$ & Adverse Events & Type of Clinical Study \\
\hline Bee venom & $\begin{array}{l}\text { Musculoskeletal } \\
\text { pain [17] }\end{array}$ & $\begin{array}{c}\text { Bee venom acupuncture } \\
\text {-concentration: } 0.05-0.5 \mathrm{mg} / \mathrm{mL} \\
-1 \text { session: } 0.01-1 \mathrm{~mL} \\
\text {-total 3-16 sessions: } 0.05-30 \mathrm{~mL}\end{array}$ & Positive & $\begin{array}{l}\text { Skin hypersensitivity } 2, \\
\text { itching } 1 \text {, pain } 2, \\
\text { pruritus } 8 \text {, burning } \\
\text { sensation } 3\end{array}$ & SR \\
\hline Bee venom & $\begin{array}{l}\text { Post-stroke shoulder } \\
\text { pain [18] }\end{array}$ & $\begin{array}{c}\text { Bee venom acupuncture } \\
\text {-concentration: } 0.1-0.5 \mathrm{mg} / \mathrm{mL} \\
\text {-1 session: } 0.1-1.5 \mathrm{~mL} \\
\text {-total 6-12 sessions: } 0.9-13.5 \mathrm{~mL}\end{array}$ & Positive & $\begin{array}{l}\text { Pain 2, pruritus } 8 \text {, } \\
\text { burning sensation } 3\end{array}$ & SR \\
\hline Bee venom & Shoulder pain [19] & $\begin{array}{c}\text { Bee venom acupuncture } \\
\text {-concentration: } 0.03-0.5 \mathrm{mg} / \mathrm{mL} \\
\text {-1 session: } 0.1-1.5 \mathrm{~mL} \\
\text {-total 6-16 sessions: } 0.6-14.8 \mathrm{~mL}\end{array}$ & Positive & $\begin{array}{l}\text { Pain 2, pruritus } 8, \\
\text { burning sensation } 3 \\
\text { Pruritus/local } \\
\text { swelling/redness 30, } \\
\text { mild, generalized } \\
\text { swelling/aching } 1\end{array}$ & SR \\
\hline
\end{tabular}

(A) Bee venom acupuncture

-concentration: $0.1 \mathrm{mg} / \mathrm{mL}$

-1 session: $0.4 \mathrm{~mL}$ (first visit), $0.6 \mathrm{~mL}$

(second visit), $0.8 \mathrm{~mL}$ (third visit),

$$
1 \mathrm{~mL} \text { (4-16 visit) }
$$

Bee venom Adhesive capsulitis [20]

-total 16 sessions: $14.8 \mathrm{~mL}$

(B) Bee venom acupuncture

-concentration: $0.03 \mathrm{mg} / \mathrm{mL}$

-1 session: $0.4 \mathrm{~mL}$ (first visit), $0.6 \mathrm{~mL}$

(second visit), $0.8 \mathrm{~mL}$ (third visit),

$1 \mathrm{~mL}$ (4-16 visit)

-total 16 sessions: $14.8 \mathrm{~mL}$

(A) Bee venom acupuncture

-concentration: $0.1 \mathrm{mg} / \mathrm{mL}$

-1 session: $0.4 \mathrm{~mL}$ (first visit), $0.6 \mathrm{~mL}$

(second visit), $0.8 \mathrm{~mL}$ (third visit),

$$
1 \mathrm{~mL} \text { (4-16 visit) }
$$

Bee venom Adhesive capsulitis [21] -total 16 sessions: $14.8 \mathrm{~mL}$

(B) Bee venom acupuncture

Positive

n.r.

RCT

-concentration: $0.03 \mathrm{mg} / \mathrm{mL}$

-1 session: $0.4 \mathrm{~mL}$ (first visit), $0.6 \mathrm{~mL}$

(second visit), $0.8 \mathrm{~mL}$ (third visit),

$$
1 \mathrm{~mL} \text { (4-16 visit) }
$$

\begin{tabular}{|c|c|c|c|c|c|}
\hline Bee venom & $\begin{array}{l}\text { Central post stroke } \\
\text { pain [22] }\end{array}$ & $\begin{array}{l}\text { Bee venom acupuncture } \\
\text {-concentration: } n . r . \\
\text { - } 1 \text { session: } 0.3 \mathrm{~mL} \\
\text {-total } 6 \text { sessions: } 1.8 \mathrm{~mL}\end{array}$ & Positive & No adverse events & $\mathrm{RCT}$ \\
\hline Bee venom & $\begin{array}{l}\text { Chronic low back } \\
\text { pain [23] }\end{array}$ & $\begin{array}{c}\text { Bee venom acupuncture } \\
\text {-concentration: } 0.05 \mathrm{mg} / \mathrm{mL} \\
\text {-1 session: } 2 \mathrm{~mL} \text { (first week), } 4 \mathrm{~mL} \\
\text { (second week), } 8 \mathrm{~mL} \text { (third week) } \\
\text {-total } 6 \text { sessions: } 28 \mathrm{~mL}\end{array}$ & Positive & $\begin{array}{c}\text { Itching/sensation } 4, \\
\text { headache } 1 \text {, generalized } \\
\text { myalgia } 1\end{array}$ & RCT \\
\hline Bee venom & $\begin{array}{l}\text { Chronic low back } \\
\text { pain [24] }\end{array}$ & $\begin{array}{c}\text { Bee venom acupuncture } \\
\text {-concentration: } 0.05 \mathrm{mg} / \mathrm{mL} \\
\text {-1 session: } 0.6 \mathrm{~mL} \\
\text {-total } 8 \text { sessions: } 4.8 \mathrm{~mL}\end{array}$ & Positive & $\begin{array}{l}\text { Itching } 15 \text {, skin flare } 5 \text {, } \\
\text { edema } 4 \text {, rash } 2\end{array}$ & $\mathrm{RCT}$ \\
\hline Bee venom & $\begin{array}{l}\text { Chronic low back } \\
\text { pain [25] }\end{array}$ & $\begin{array}{l}\text { Bee venom acupuncture } \\
\text {-concentration: } 0.3 \mathrm{mg} / \mathrm{mL} \\
\text { - } 1 \text { session: } 0.1 \mathrm{~mL} \\
\text {-total } 5 \text { sessions: } 0.5 \mathrm{~mL}\end{array}$ & Positive & Skin hypersensitivity 1 & RCT \\
\hline Bee venom & $\begin{array}{l}\text { Delayed onset muscle } \\
\text { soreness [26] }\end{array}$ & $\begin{array}{c}\text { Bee venom gel } \\
\text {-concentration: ultrasound gel and } 1 \\
\mathrm{mg} / \mathrm{mL} \text { bee venom mixed at a ratio of } \\
9: 1 \\
-1 \text { session: } n . r . \\
\text {-total 3 session: } \text { n.r. }\end{array}$ & Positive & n.r. & RCT \\
\hline Bee venom & $\begin{array}{l}\text { Herniated lumbar } \\
\text { disc [27] }\end{array}$ & $\begin{array}{l}\text { Bee venom acupuncture } \\
\text {-concentration: } 0.05-0.4 \mathrm{mg} / \mathrm{mL} \\
-1 \text { session: } 0.01-0.15 \mathrm{~mL} \\
\text {-total } 7 \text { sessions: } 0.07-1.05 \mathrm{~mL}\end{array}$ & Positive & n.r. & RCT \\
\hline Bee venom & $\begin{array}{l}\text { Herniated lumbar } \\
\text { disc [28] }\end{array}$ & $\begin{array}{c}\text { Bee venom acupuncture } \\
\text {-concentration: } 0.25 \mathrm{mg} / \mathrm{mL} \text { (early } \\
\text { stage) }-0.5 \mathrm{mg} / \mathrm{mL} \text { (last stage) } \\
\text {-1 session: } 0.25 \mathrm{mg} / \mathrm{mL} \text { bee venom } \\
0.1 \mathrm{~mL}, 0.5 \mathrm{mg} / \mathrm{mL} \text { bee venom } 1 \mathrm{~mL} \\
\text {-total } 7 \text { sessions: } 0.25 \mathrm{mg} / \mathrm{mL} \text { bee } \\
\text { venom } 3 \mathrm{~mL}, 0.5 \mathrm{mg} / \mathrm{mL} \text { bee venom } \\
30 \mathrm{~mL}\end{array}$ & Positive & n.r. & RCT \\
\hline
\end{tabular}

-total 16 sessions: $14.8 \mathrm{~mL}$ 
Table 2. Cont.

\begin{tabular}{|c|c|c|c|c|c|}
\hline $\begin{array}{c}\text { Type of Animal } \\
\text { Venom }\end{array}$ & $\begin{array}{l}\text { Treatment } \\
\text { Diseases }\end{array}$ & $\begin{array}{c}\text { Intervention } \\
\text { (Concentration, Treatment Sessions, } \\
\text { Amount of Venom Use) }\end{array}$ & $\begin{array}{l}\text { Clinical } \\
\text { Effects }\end{array}$ & Adverse Events & Type of Clinical Study \\
\hline Bee venom & Knee osteoarthritis [29] & $\begin{array}{l}\text { Bee venom acupuncture } \\
\text {-concentration: } 0.3 \mathrm{mg} / \mathrm{mL} \\
\text { - } 1 \text { session: } 0.5-1 \mathrm{~mL} \\
\text {-total } 8 \text { sessions: } 4-8 \mathrm{~mL}\end{array}$ & Positive & n.r. & $\mathrm{RCT}$ \\
\hline Bee venom & knee osteoarthritis [30] & $\begin{array}{c}\text { Bee venom acupuncture } \\
\text {-concentration: bee venom powder } \\
1 \mathrm{mg} \text { and } 1 \mathrm{~mL} 0.5 \% \text { lidocaine were } \\
\text { mixed } \\
\text {-1 session: } 1.2 \mathrm{~mL}(1-3 \text { weeks }), 1.5 \mathrm{~mL} \\
(4-12) \\
\text {-total } 12 \text { sessions: } 17.1 \mathrm{~mL}\end{array}$ & Positive & n.r. & RCT \\
\hline Bee venom & Multiple sclerosis [31] & $\begin{array}{l}\text { Bee venom sting therapy } \\
\text {-concentration: bee sting } \\
\text {-1 session: maximum } 20 \text { times } \\
\text {-total } 72 \text { sessions: maximum } \\
1440 \text { times }\end{array}$ & Positive & $\begin{array}{c}\text { Extreme swelling 2, } \\
\text { itching } 4 \text {, flulike } \\
\text { symptoms 5, local } \\
\text { tender- } \\
\text { ness/swelling/redness } \\
\text { n.r. }\end{array}$ & $\mathrm{RCT}$ \\
\hline Bee venom & Neck pain [32] & $\begin{array}{l}\text { Bee venom acupuncture } \\
\text {-concentration: } 0.3 \mathrm{mg} / \mathrm{mL} \\
-1 \text { session: } 0.1 \mathrm{~mL} \\
\text {-total: } \text { n.r. }\end{array}$ & Positive & Skin hypersensitivity 1 & $\mathrm{RCT}$ \\
\hline Bee venom & $\begin{array}{l}\text { Pelvic inflammatory } \\
\text { disease [33] }\end{array}$ & $\begin{array}{c}\text { Bee venom gel } \\
\text {-concentration: bee venom } 20 \mu \mathrm{g} / \mathrm{gel} \\
1 \mathrm{~g} \\
-1 \text { session: n.r. } \\
\text {-total } 12 \text { sessions: n.r. }\end{array}$ & Positive & n.r. & $\mathrm{RCT}$ \\
\hline Bee venom & $\begin{array}{c}\text { Temporomandibular } \\
\text { disorder [34] }\end{array}$ & $\begin{array}{l}\text { Bee venom ointment } \\
\text {-concentration: } 0.0005 \% \\
-1 \text { time: } \text { n.r. } \\
\text {-total } 42 \text { times: } \text { n.r. }\end{array}$ & Positive & n.r. & $\mathrm{RCT}$ \\
\hline Bee venom & Wrist sprain [35] & $\begin{array}{l}\text { Bee venom acupuncture } \\
\text {-concentration: } 0.3 \mathrm{mg} / \mathrm{mL} \\
\text {-1 session: } 0.05-10.5 \mathrm{~mL} \\
\text {-over } 2 \text { sessions: n.r. }\end{array}$ & Positive & n.r. & $\mathrm{RCT}$ \\
\hline Bee venom & $\begin{array}{l}\text { Polycystic ovary } \\
\text { syndrome [36] }\end{array}$ & $\begin{array}{c}\text { Bee venom gel } \\
\text {-concentration: } \text { n.r. } \\
\text {-1 session: } 30-50 \mathrm{~g} \\
\text {-total } 28 \text { sessions: } 840-1400 \mathrm{~g}\end{array}$ & NS & n.r. & $\mathrm{RCT}$ \\
\hline Bee venom & Acute ankle sprain [37] & $\begin{array}{l}\text { Bee venom acupuncture } \\
\text {-concentration: } 0.3 \mathrm{mg} / \mathrm{mL} \\
-1 \text { session: } 0.06 \mathrm{~mL} \\
\text {-total } 7 \text { sessions: } 0.42 \mathrm{~mL}\end{array}$ & Positive & Itching 1 & $\mathrm{RCT}$ \\
\hline Bee venom & Acute ankle sprain [38] & $\begin{array}{c}\text { Bee venom acupuncture } \\
\text {-concentration: } 0.25 \mathrm{mg} / \mathrm{mL} \\
0.1 \mathrm{mg} / \mathrm{mL} \\
\text {-1 session: } 0.25 \mathrm{mg} / \mathrm{mL} 0.3 \mathrm{~mL} \\
0.1 \mathrm{mg} / \mathrm{mL} 0.2 \mathrm{~mL} \\
\text {-total } 3 \text { sessions: } 0.25 \mathrm{mg} / \mathrm{mL} 0.9 \mathrm{~mL} \text {, } \\
0.1 \mathrm{mg} / \mathrm{mL} 0.6 \mathrm{~mL}\end{array}$ & NS & n.r. & $\mathrm{RCT}$ \\
\hline Bee venom & Parkinson's disease [39] & $\begin{array}{c}\text { Bee venom acupuncture } \\
\text {-concentration: } 0.05 \mathrm{mg} / \mathrm{mL} \\
-1 \text { session: } 1 \mathrm{~mL} \\
\text {-total } 16 \text { sessions: } 16 \mathrm{~mL}\end{array}$ & Positive & Itching 1 & $\mathrm{RCT}$ \\
\hline Bee venom & Parkinson's disease [40] & $\begin{array}{c}\text { Bee venom acupuncture } \\
\text {-concentration: bee venom powder } \\
1 \mathrm{mg} \text { and } 20 \mathrm{~mL} \text { normal saline were } \\
\text { mixed } \\
-1 \text { session: } 1 \mathrm{~mL} \\
\text {-total } 24 \text { sessions: } 24 \mathrm{~mL}\end{array}$ & Positive & $\begin{array}{c}\text { Mild pain/slight } \\
\text { bleeding n.r., mild } \\
\text { itching/mild swelling } \\
\text { n.r. }\end{array}$ & $\mathrm{RCT}$ \\
\hline Bee venom & Parkinson's disease [41] & $\begin{array}{c}\text { Bee venom acupuncture } \\
\text {-concentration: bee venom } 0.1 \mathrm{mg} \text { and } \\
1 \mathrm{~mL} \text { of } \mathrm{NaCl} 0.9 \% \text { were mixed } \\
-1 \text { session: } 0.05 \mathrm{~mL} \\
\text {-total } 11 \text { sessions: } 0.55 \mathrm{~mL}\end{array}$ & NS & $\begin{array}{c}\text { Redness /itching n.r., } \\
\text { insomnia 1, nausea 3, } \\
\text { fatigue } 2 \text {, dyskinesia 1, } \\
\text { bee venom specific IgE } \\
\text { 18, bee venom specific } \\
\text { IgG4 } 12\end{array}$ & $\mathrm{RCT}$ \\
\hline Bee venom & $\begin{array}{l}\text { Post-stroke shoulder } \\
\text { pain [42] }\end{array}$ & $\begin{array}{l}\text { Bee venom acupuncture } \\
\text {-concentration: n.r. } \\
\text {-1 session: } 0.3-0.6 \mathrm{~mL} \\
\text {-total } 12 \text { sessions: } 3.6-7.2 \mathrm{~mL}\end{array}$ & Positive & n.r. & $\mathrm{RCT}$ \\
\hline
\end{tabular}


Table 2. Cont.

\begin{tabular}{|c|c|c|c|c|c|}
\hline $\begin{array}{l}\text { Type of Animal } \\
\text { Venom }\end{array}$ & $\begin{array}{l}\text { Treatment } \\
\text { Diseases }\end{array}$ & $\begin{array}{c}\text { Intervention } \\
\text { (Concentration, Treatment Sessions, } \\
\text { Amount of Venom Use) }\end{array}$ & $\begin{array}{l}\text { Clinical } \\
\text { Effects }\end{array}$ & Adverse Events & Type of Clinical Study \\
\hline Bee venom & $\begin{array}{l}\text { Post stroke shoulder } \\
\text { pain [43] }\end{array}$ & $\begin{array}{l}\text { Bee venom acupuncture } \\
\text {-concentration: } 0.1 \mathrm{mg} / \mathrm{mL} \\
-1 \text { session: } 0.6 \mathrm{~mL} \\
\text {-total } 6 \text { sessions: } 3.6 \mathrm{~mL}\end{array}$ & Positive & $\begin{array}{l}\text { Pain 2, pruritus } 8 \text {, } \\
\text { burning sensation } 3\end{array}$ & RCT \\
\hline Bee venom & $\begin{array}{l}\text { Post-stroke shoulder } \\
\text { pain [44] }\end{array}$ & $\begin{array}{l}\text { Bee venom acupuncture } \\
\text {-concentration: } 0.5 \mathrm{mg} / \mathrm{mL} \\
-1 \text { session: } 0.25-0.5 \mathrm{~mL} \\
\text {-total } 12 \text { sessions: } 3-6 \mathrm{~mL}\end{array}$ & Positive & n.r. & RCT \\
\hline Bee venom & $\begin{array}{l}\text { Post-stroke shoulder } \\
\text { pain [45] }\end{array}$ & $\begin{array}{c}\text { Bee venom acupuncture } \\
\text {-concentration: } 0.1-0.25 \mathrm{mg} / \mathrm{mL} \\
\text {-1 session: } 0.1-1.5 \mathrm{~mL} \\
\text {-total } 9 \text { sessions: } 0.9-13.5 \mathrm{~mL}\end{array}$ & Positive & n.r. & RCT \\
\hline Bee venom & $\begin{array}{l}\text { Post-stroke shoulder } \\
\text { pain [46] }\end{array}$ & $\begin{array}{c}\text { Bee venom acupuncture } \\
\text {-concentration: } 0.05 \mathrm{mg} / \mathrm{mL} \\
\text { - } 1 \text { session: } 0.1 \mathrm{~mL} \\
\text {-total } 6 \text { sessions: } 0.6 \mathrm{~mL}\end{array}$ & NS & n.r. & RCT \\
\hline Bee venom & $\begin{array}{l}\text { Rheumatoid } \\
\text { arthritis [47] }\end{array}$ & $\begin{array}{l}\text { Bee venom acupuncture } \\
\text {-concentration: } 0.3 \mathrm{mg} / \mathrm{mL} \\
\text {-1 session: } 0.2-1.0 \mathrm{~mL} \\
\text {-total } 16 \text { sessions: } 3.2-16 \mathrm{~mL}\end{array}$ & Positive & n.r. & RCT \\
\hline Bee venom & $\begin{array}{l}\text { Rheumatoid } \\
\text { arthritis [48] }\end{array}$ & $\begin{array}{l}\text { Bee venom sting therapy } \\
\text {-concentration: bee sting } \\
-1 \text { session: } 5-15 \text { times } \\
\text {-total } 24 \text { sessions: } 120-360 \text { times }\end{array}$ & NS & $\begin{array}{c}\text { Itching } 60, \text { redness } 44, \\
\text { swelling } 41 \text {, burning } \\
\text { sensation } 9, \text { lymph node } \\
\text { hypertrophy } 2\end{array}$ & RCT \\
\hline Snake venom & $\begin{array}{l}\text { Acute ischemic } \\
\text { stroke [49] }\end{array}$ & $\begin{array}{c}\text { Snake venom intravenous injection } \\
\text {-concentration: } 0.5-1 \mathrm{U} / \mathrm{kg} \\
-1 \text { day: } 0.5-1 \mathrm{U} / \mathrm{kg} \text { then variable dose } \\
\text { to keep fibrinogen level } \\
70-130 \mathrm{mg} / \mathrm{dL} \\
\text {-total } 7-14 \text { days }\end{array}$ & Positive & n.r. & SR \\
\hline Snake venom & $\begin{array}{l}\text { Haemorrhage control in } \\
\text { bi-maxillary } \\
\text { orthognathic } \\
\text { surgery [50] }\end{array}$ & $\begin{array}{l}\text { Snake venom intravenous injection } \\
\text {-concentration: } 1 \mathrm{U} / \mathrm{mL} \text { aqueous } \\
\text { solution containing } 0.9 \% \text { sodium } \\
\text { chloride, } 0.3 \% \text { phenol, and water for } \\
\text { injection } \\
\text {-1session: n.r. (single bolus dose } \\
\text { before surgery) }\end{array}$ & Positive & No adverse events & $\mathrm{RCT}$ \\
\hline Snake venom & $\begin{array}{l}\text { Haemorrhage control in } \\
\text { fracture-related hip } \\
\text { hemiarthroplasty [51] }\end{array}$ & $\begin{array}{l}\text { Snake venom intravenous injection } \\
\text {-concentration: n.r. } \\
\text {-1 session: } 1 \mathrm{U} \\
\text {-total } 3 \text { sessions: } 3 \mathrm{U}, \text { before surgery } \\
\text { (10-15 h preoperative) and repeated in } \\
\text { equivalent volumes at } 30 \text { min } \\
\text { preoperative and } 12 \mathrm{~h} \text { postoperative }\end{array}$ & Positive & No adverse events & RCT \\
\hline Snake venom & $\begin{array}{l}\text { Acute myocardial } \\
\text { infarction [52] }\end{array}$ & $\begin{array}{l}\text { Snake venom intravenous injection } \\
\text {-concentration: } 7.5 \mathrm{U} / 100 \mathrm{~mL} \text { glucose } \\
\text { solution } \\
\text {-1 session: } 7.5 \mathrm{U} \text { snake venom was } \\
\text { placed in a } 100 \mathrm{~mL} 5 \% \text { glucose } \\
\text { solution, and reduced to } 2.5 \mathrm{U} \\
\text {-total } 2-14 \text { sessions }\end{array}$ & Positive & n.r. & $\mathrm{RCT}$ \\
\hline Snake venom & $\begin{array}{l}\text { Acute myocardial } \\
\text { infarction [53] }\end{array}$ & $\begin{array}{l}\text { Snake venom intracoronary or } \\
\text { intravenous injection } \\
\text {-concentration: n.r. } \\
\text {-1 session: n.r. (single bolus dose } \\
\text { before surgery) }\end{array}$ & Positive & n.r. & RCT \\
\hline Snake venom & $\begin{array}{l}\text { Haemostasis during } \\
\text { transvesical prostatic } \\
\text { adenomectomy [54] }\end{array}$ & $\begin{array}{l}\text { Snake venom intravenous injection } \\
\text {-concentration: n.r. } \\
\text {-1session: } 1 \text { ample } \\
\text {-total } 14 \text { sessions: } 14 \text { samples }\end{array}$ & Positive & n.r. & $\mathrm{RCT}$ \\
\hline Snake venom & $\begin{array}{l}\text { Acute cerebral } \\
\text { infarction [55] }\end{array}$ & $\begin{array}{l}\text { Snake venom intravenous injection } \\
\text {-concentration: } 0.0005 \mathrm{U} / \mathrm{kg} \\
\text { - } 1 \text { day: } 0.0005 \mathrm{U} / \mathrm{kg} \times 2 \mathrm{times} / \text { day } \\
\text {-total } 7 \text { days: } 0.007 \mathrm{U} / \mathrm{kg}\end{array}$ & Positive & No adverse events & RCT \\
\hline Toad venom & Cancer-related pain [56] & $\begin{array}{l}\text { Toad venom intravenous injection } \\
\text {-concentration: n.r. } \\
\quad-1 \text { session: n.r. } \\
\text {-total } 1 \text { week-30 days sessions: n.r. }\end{array}$ & Positive & n.r. & SR \\
\hline
\end{tabular}


Table 2. Cont.

\begin{tabular}{|c|c|c|c|c|c|}
\hline $\begin{array}{l}\text { Type of Animal } \\
\text { Venom }\end{array}$ & $\begin{array}{l}\text { Treatment } \\
\text { Diseases }\end{array}$ & $\begin{array}{c}\text { Intervention } \\
\text { (Concentration, Treatment Sessions, } \\
\text { Amount of Venom Use) }\end{array}$ & $\begin{array}{l}\text { Clinical } \\
\text { Effects }\end{array}$ & Adverse Events & Type of Clinical Study \\
\hline Toad venom & Gastric cancer [57] & $\begin{array}{l}\text { Toad venom intravenous injection } \\
\text {-concentration: } \text { n.r. } \\
\text {-1 session: } 10-50 \mathrm{~mL} \\
\text {-total } 28-112 \text { days: n.r. }\end{array}$ & Positive & $\begin{array}{l}\text { Leukopenia } 113, \\
\text { nausea/vomiting } 56, \\
\text { diarrhea } 28\end{array}$ & SR \\
\hline Toad venom & Liver cancer [58] & $\begin{array}{l}\text { Toad venom intravenous injection } \\
\text {-concentration: n.r. } \\
\text {-1 session: } 10-30 \mathrm{~mL} \\
\text {-total sessions: n.r. }\end{array}$ & Positive & Nausea/vomiting 2 & $\begin{array}{c}\text { SR } \\
\text { (network meta-analysis) }\end{array}$ \\
\hline Toad venom & $\begin{array}{l}\text { Metastatic bone } \\
\text { tumors [59] }\end{array}$ & $\begin{array}{l}\text { Toad venom capsule } \\
\text {-concentration: n.r. } \\
\text {-1 session: } 500 \mathrm{mg} \\
\text {-total } 36 \text { sessions: } 18 \mathrm{~g}\end{array}$ & Positive & $\begin{array}{l}\text { Fever } 3 \text {, nausea and } \\
\text { vomiting } 4 \text {, diarrhea } 2, \\
\text { muscle soreness } 3\end{array}$ & $\mathrm{RCT}$ \\
\hline Toad venom & $\begin{array}{l}\text { Primary liver } \\
\text { cancer [60] }\end{array}$ & $\begin{array}{l}\text { Toad venom intravenous injection } \\
\text {-concentration: n.r. } \\
\text {-1 session: } 20 \mathrm{~mL} \text { toad venom was } \\
\text { placed in a } 250 \mathrm{~mL} 5 \% \text { glucose } \\
\text { solution } \\
\text {-total } 15 \text { session: } 300 \mathrm{~mL}(20 \mathrm{~mL} \text { toad } \\
\text { venom was placed in a } 250 \mathrm{~mL} 5 \% \\
\text { glucose solution } \times 15 \text { times })\end{array}$ & Positive & n.r. & $\mathrm{RCT}$ \\
\hline Toad venom & $\begin{array}{l}\text { Advanced pancreatic } \\
\text { adenocarcinomas [61] }\end{array}$ & $\begin{array}{l}\text { Toad venom injection } \\
\text {-concentration: } \\
\text {-1session: } 20 \mathrm{~mL} \mathrm{~m}^{-2} \text { over } 2 \mathrm{~h} \\
\text {-total } 15 \text { sessions: } 300 \mathrm{~mL} \mathrm{~m}^{-2}\end{array}$ & NS & n.r. & $\mathrm{RCT}$ \\
\hline
\end{tabular}

n.r.: not reported; NS: not statistically significant effectiveness between groups; Positive: statistically significant effectiveness between groups; RCT: randomized controlled trials; SR: systematic review.

\section{Discussion}

To our knowledge, this study is the first to report on the three animal venom therapies used in KM institutions in South Korea. It covers the current status of the production of pharmacopuncture medicines based on animal venoms, their production processes, and indications. The government played a leading role in this study, which aimed to understand the current status of the production of animal venom pharmacopuncture medicines in EHDs. All of the pharmacopuncture medicines used in KM institutions are being produced in EHDs; this study covered 11 of the 16 EHDs that produced pharmacopuncture medicines (68.8\%). Since EHDs prepared these medicines following the prescriptions issued by KM institutions, the production records at EHDs can be considered to represent the current status of the pharmacopuncture medicines used in KM institutions. Therefore, this study provides an overall status of the use of animal venoms for therapeutic applications by KM institutions.

Bee venom products represent the second-largest quantity of pharmacopuncture medicines in South Korea, accounting for $99.06 \%$ of the total animal venom-based pharmacopuncture. While bee venom causes neurotoxic symptoms including local paresthesias, headache, dizziness, nausea and vomiting, muscle aches, and rarely, cerebrovascular infarcts [62-65], recent non-clinical studies demonstrated that bee venom has antiinflammatory [66], anti-nociceptive [67], and anti-cancer activities [68]. The toxic reactions of bee venom therapy in clinical studies ranged from fatigue [69], erythematous plaques [70], pallor face [71], nausea [71], vomiting [71], and other minor side effects to more serious ones such as limb paralysis [72], dyspnea [72], unconsciousness [73], and death [73]. There have been many clinical and non-clinical studies reporting the side effects and benefits of bee venom therapy. However, due to the possibility of variation in the species of bees and the nutrients they take, the composition of bee venom can differ depending on geography. Therefore, it is necessary to standardize the material, evaluate its toxicity, and examine its benefits.

Snake venom is the 13th most frequently used pharmacopuncture medicine in KM institutions. While snake venoms can be cardiotoxic (e.g., arrhythmias, bradycardia, tachycardia and hypotension) [74], myotoxic (e.g., myalgias, myopathy and rhabdomyolysis) [74], neurotoxic (e.g., ptosis, external ophthalmoplegia, dysphagia, dysphonia and 
broken neck sign) [75], and nephrotoxic (e.g., ischemia and renal failure) [74], non-clinical studies of snake venom reported effects such as a decrease in blood pressure [76], regulation of blood coagulation [77], and anti-tumor [78] and analgesic activities [78]. The snakevenom-based medicines that have been approved by the Food and Drug Administration (FDA) are administered using tablets (captopril) or intravenous injections (eptifibatide and tirofiban) [79]. Further studies are needed to evaluate the efficacy and toxicity of the routes of administration (e.g., oral, muscle injection, or infusion, etc.).

Toad venom is the least frequently used animal venom in KM institutions $(0.27 \%)$. While toad venom has toxic effects and causes gastrointestinal, mental, cardiac conduction, and arrhythmic disturbances [80], non-clinical studies have reported anti-inflammatory [81], antiplasmodial activity [82], and antiproliferative effects on cancer cells [83]. The treatment toxicity is the most important factor limiting its use [84]. Further studies should be carried out to evaluate the benefits and side effects of its use to treat inflammatory diseases.

As is the case with bee stings, animal venom was used as it was, in some cases. However, in most cases, it was used as diluted to an appropriate concentration for clinical purposes. Animal venoms may cause idiosyncratic reactions depending on the individual in addition to their toxic effect [85]. For this reason, it is recommended to have the patient take a certain amount before clinical applications, in order to determine whether the use or administration of such a substance causes adverse effects or not. Also, according to the analysis on the 1,192,667 cases of accidents caused by animal venom over 12 years in Brazil by Chippaux [86], snake bites were linked to the highest fatality rate of $0.43 \%$, followed by bee stings, of which the fatality rate was $0.33 \%$. Since animal venom has toxic effects, it is necessary to refine, dilute, or otherwise process it before clinical use.

This study has several limitations. First, this study was a literature review of the indications and benefits of pharmacopuncture medicines based on animal venoms, without any reviews on the clinical indications in the real world. Further surveys of KM doctors are needed to cover the clinical indications and side effects. Second, a literature review of indications and side effects used only articles published in South Korea, and only articles dealing with injectables were reviewed. International databases will need to be reviewed to evaluate animal venoms factors such as the species, extraction process, and administration routes. Third, this survey was conducted by the government, which measured its accuracy and trustworthiness. However, the validity of the questionnaire was not confirmed.

Despite these limitations, this is the first study reviewing the production volume and current status of animal venom-based pharmacopuncture medicines used in clinical practice in South Korea. Collecting further data is necessary for the standardization of pharmacopuncture medicine and safety of dosages and clinical applications.

\section{Conclusions}

This study reports that bee, snake, and toad animal venoms are being used in medical institutions and have the potential for drug development. Based on the clinical evidence for animal venom summarized in this study, it is expected that the development of therapeutic agents for target diseases will be conducted.

\section{Methods}

\subsection{Overview of the Study}

A survey of community health centers across the country was conducted with the cooperation of the Ministry of Health and Welfare to understand the nationwide status of the production and use of animal venom medicinal products in the country.

For this purpose, a systematically developed questionnaire was distributed to participating organizations. The process of producing animal venom-based pharmacopuncture medicines was analyzed using textbooks, research papers, and data from the EHDs currently producing such medicines. 


\subsection{Study Sample}

The study sample consisted of 256 community health centers across the country that were responsible for establishing and supervising EHDs in South Korea.

\subsection{Questionnaire Development and Distribution}

To develop the questionnaire, a group of five experts was formed (including two KM specialists with an average of $10+$ years of clinical experience of pharmacopuncture (J.K.P., S.B.C.), one PhD with $10+$ years of experience in the KM field (G.L.), one expert in the GMP, and one expert in hazard analysis critical control point (HACCP)). The draft of the questionnaire was developed based on previous studies [87-89]. The final version of the questionnaire was agreed upon through a review and revision process (Additional file 2 in Supplementary Materials).

To ensure nationwide representation, the questionnaire was sent together with official letters to 256 community health centers in South Korea through the Ministry of Health and Welfare over the period from 1 through 31 October 2019. A community health center has the authority to approve the establishment and supervision of an EHD. The centers were asked to complete the questionnaire based on the status in 2018; the community health centers were also instructed to complete the questionnaire only when their EHD produced pharmacopuncture medicines. To enhance the accuracy of the survey, it was requested that the questionnaire be completed by the KM pharmacist or the head of the KM institution that established the EHD.

\subsection{Questionnaire Items}

To obtain accurate information on the use of animal venoms in pharmacopuncture, the survey items consisted of two items concerning the basic status of EHDs (i.e., the type of the medical institution that set up the EHD and the location) and three items relating the production of pharmacopuncture medicines (the type of pharmacopuncture, herbal ingredients, and production volume). The questionnaire was designed so that the five items could be completed in a Microsoft Office Excel file (verson 16.0, 2013, Microsoft ${ }^{\circledR}$, Redmond, WA, USA).

\subsection{Data Collection}

Completed questionnaires that were sent back to us from the Ministry of Health and Welfare were reviewed for the integrity of the completed data in Excel files and sorted out by the relevant items. For any missing or incomplete data, the EHD was called or contacted via email to request additional or missing data and correct the incorrect data as much as possible.

\subsection{Data Analysis}

The data gathered using the questionnaire were processed using SPSS version 21.0 (IBM, Armonk, NY, USA). An explorative data analysis (EDA) was conducted. A summary of the data was prepared including the average, standard deviations, and frequency $(n, \%)$. Any incomplete data were treated as missing.

\subsection{Literature Search of the Production Process and Clinical Effectiveness of Animal Venom Medications}

The survey identified 46 types of pharmacopuncture medicines; three animal venoms (bee, snake, and toad venoms) were selected by the reviewing experts. The external experts were a professor of KM university who is a pharmaceutical board member at the national hospital, a KM professor who majored in herbal medicines, a general manager of an EHD with 8+ years of experience in preparing pharmacopuncture, and a quality control (QC) team leader from a pharmaceutical company that manufactures herbal drugs.

The researcher also reviewed textbooks used in South Korean medical schools for pharmacopuncture and related academic papers to review the production process and 
indications of the three selected animal venoms. The following electronic databases using keywords "bee venom", "snake venom", and "toad venom" were searched to identify relevant studies for inclusion in the review from inception to September 2020: Pubmed, EMBASE, Medline, Cochrane library, and six Korean databases (National Digital Science Library (NDSL), the Korean Traditional Knowledge Portal, KoreaMed, the Oriental Medicine Advanced Searching Integrated System (OASIS), the Research Information Sharing Service (RISS), and The National Library of Korea). All identified clinical studies and systematic reviews were examined to understand the clinical indications and effects of animal venom-based therapy. Two researchers (S.S.H. and J.E.H.) independently reviewed and screened the titles and abstracts of the retrieved studies based on predefined eligibility criteria. Two independent reviewers (J.W.K. and B.C.S.) extracted the treated diseases and the effectiveness of animal venom-based therapy. The systematic review reflected the results of the meta-analysis.

Supplementary Materials: The following are available online at https://www.mdpi.com/2072-6 651/13/2/105/s1, Additional file 1: Demographics of pharmacopuncture-EHDs, Additional file 2: Current status of external herbal dispensaries preparing pharmacopuncture: a survey.

Author Contributions: Conceptualization, S.-H.S., B.-C.S. and G.L.; methodology, B.-C.S. and G.L.; writing-original draft, S.-H.S., J.-W.K. and J.-E.H.; writing—review and editing, B.-C.S., G.L. and J.-K.P.; visualization, J.-W.K.; supervision, G.L. All authors have read and agreed to the published version of the manuscript.

Funding: This research was supported by a grant from the Project of National Development Institute of Korean Medicine, Accreditation of External Herbal Dispensaries of Traditional Korean Medicine Clinics, funded by the Korean Ministry of Health and Welfare.

Institutional Review Board Statement: Ethical review and approval were waived for this study, because this study did not include personal information and was for evaluating public services.

Informed Consent Statement: Not applicable.

Data Availability Statement: The data will be made available upon reasonable request.

Acknowledgments: We would like to thank the external herbal dispensary of Jaseng Hospital (herb.jaseng.co.kr/) for providing a picture of the pharmacopuncture preparation process.

Conflicts of Interest: The authors declare no conflict of interest.

\section{References}

1. Utkin, Y.N. Animal venom studies: Current benefits and future developments. World J. Biol. Chem. 2015, 6, 28-33. [CrossRef]

2. Gwaltney-Brant, S.M. Chapter 49-Zootoxins—Reproductive and Developmental Toxicology, 2nd ed.; Academic Press: Cambridge, MA, USA, 2017.

3. Hakim, M.A.; Yang, S.; Lai, R. Centipede Venoms and Their Components: Resources for Potential Therapeutic Applications. Toxins 2015, 7, 4832-4851. [CrossRef] [PubMed]

4. Chen, N.; Xu, S.; Zhang, Y.; Wang, F. Animal protein toxins: Origins and therapeutic applications. Biophys. Rep. 2018, 4, 233-242. [CrossRef] [PubMed]

5. Jang, S.B.; Kim, K.H. Clinical effectiveness and adverse events of bee venom therapy: A systematic review of randomized controlled trials. Toxins 2020, 12, 558. [CrossRef]

6. El-Aziz, M.A.T.; Soares, A.G.; Stockand, J.D. Snake venoms in drug discovery: Valuable therapeutic tools for life saving. Toxins 2019, 11, 564. [CrossRef] [PubMed]

7. de Souza, J.M.; Goncalves, B.D.C.; Gomez, M.V.; Vieira, L.B.; Ribeiro, F.M. Animal toxins as therapeutic tools to treat neurodegenerative diseases. Front. Pharmacol. 2018, 9, 145. [CrossRef] [PubMed]

8. Yoon, J.M.; Kim, K.H.; Oh, Y.T.; Kim, J.W.; Yook, T.H. The Analysis of the Recent Research Trend of Pharmacopuncture. J. Soc. Prev. Korean Med. 2018, 22, 55-63.

9. Park, J.; Lee, H.; Shin, B.C.; Lee, M.S.; Kim, B.; Kim, J.I. Pharmacopuncture in Korea: A systematic review and meta-analysis of randomized controlled trials. Evid. Based Complement. Alternat. Med. 2016, 2016, 4683121. [CrossRef]

10. Sung, S.H.; Shin, B.C.; Park, M.J.; Kim, K.H.; Kim, J.W.; Ryu, J.Y.; Park, J.K. Current status of management on pharmacopuncture in Korea through introduction of an accreditation system. J. Pharmacopunct. 2019, 22, 75-82.

11. Kim, D.H.; Cho, S.J.; Ko, J.A. Policy Improvement Plan Based on Korean Medicine Use; Health Insurance Review \& Assessment Service: Wonju, Korea, 2015. 
12. Ministry of Health and Welfare. Study on Methods to Prove Reasonable Safety and Efficacy of Herbal Medicines; Ministry of Health and Welfare: Sejong, Korea, 2017.

13. Ministry of Health and Welfare, National Development Institute of Korean Medicine, Gallup Korea. 2017 Years National Survey for Usage and Consumption of Traditional Herbal Medicine; National Development Institute of Korean Medicine: Seoul, Korea, 2018.

14. Kim, J.H.; Kim, Y.K. A study on the facility standard of herbal dispensaries. J. Korean Med. 2017, 38, 81-92. [CrossRef]

15. Korean Pharmacopuncture Institute. Pharmacopuncturology; Elsevier Korea: Seoul, Korea, 2011.

16. Hayes, W.K.; Fox, G.A.; Nelsen, D.R. Venom collection from spiders and snakes: Voluntary and involuntary extractions ("Milking”) and venom gland extractions. Methods Mol. Biol. 2020, 2068, 53-71. [PubMed]

17. Lee, M.S.; Pittler, M.H.; Shin, B.C.; Kong, J.C.; Ernst, E. Bee venom acupuncture for musculoskeletal pain: A review. J. Pain 2008, 9, 289-297. [CrossRef] [PubMed]

18. Lim, S.M.; Lee, S.H. Effectiveness of bee venom acupuncture in alleviating post-stroke shoulder pain: A systematic review and meta-analysis. J. Integr. Med. 2015, 13, 241-247. [CrossRef]

19. Shen, L.; Lee, J.H.; Joo, J.C.; Park, S.J.; Song, Y.S. Bee venom acupuncture for shoulder pain: A systematic review and meta-analysis of randomized controlled trials. J. Pharmacopunct. 2020, 23, 44-53.

20. Koh, P.S.; Seo, B.K.; Cho, N.S.; Park, H.S.; Park, D.S.; Baek, Y.H. Clinical effectiveness of bee venom acupuncture and physiotherapy in the treatment of adhesive capsulitis: A randomized controlled trial. J. Shoulder Elb. Surg. 2013, 22, 1053-1062. [CrossRef] [PubMed]

21. Park, Y.C.; Koh, P.S.; Seo, B.K.; Lee, J.W.; Cho, N.S.; Park, H.S.; Park, D.S.; Baek, Y.H. Long-term effectiveness of bee venom acupuncture and physiotherapy in the treatment of adhesive capsulitis: A one-year follow-up analysis of a previous randomized controlled trial. J. Altern. Complement. Med. 2014, 20, 919-924. [CrossRef]

22. Cho, S.Y.; Park, J.Y.; Jung, W.S.; Moon, S.K.; Park, J.M.; Ko, C.N.; Park, S.U. Bee venom acupuncture point injection for central post stroke pain: A preliminary single-blind randomized controlled trial. Complement. Ther. Med. 2013, 21, 155-157. [CrossRef]

23. Seo, B.K.; Han, K.; Kwon, O.; Jo, D.J.; Lee, J.H. Efficacy of bee venom acupuncture for chronic low back pain: A randomized, double-blinded, sham-controlled trial. Toxins 2017, 9, 361. [CrossRef]

24. Shin, B.C.; Kong, J.C.; Park, T.Y.; Yang, C.Y.; Kang, K.W.; Choi, S.M. Bee venom acupuncture for chronic low back pain: A randomised, sham-controlled, triple-blind clinical trial. Eur. J. Integr. Med. 2012, 4, e271-e280. [CrossRef]

25. Kim, K.T.; Song, H.S. The effectiveness of bee venom acupuncture therapy on the treatment of sprain of L-spine (A randomized controlled trial: Fouble blinding). J. Korean Acupunct. Moxibustion Soc. 2005, 22, 113-120.

26. Kim, S.K.; Kim, M.C. The affect on delayed onset muscle soreness recovery for ultrasound with bee venom. J. Phys. Ther. Sci 2014, 26, 1419-1421. [CrossRef] [PubMed]

27. Chung, W.S.; Lee, J.S.; Chung, S.H.; Kim, S.S. The effect of bee venom acupuncture on patient with hernation of nucleus pulposus of lumbar spine. J. Orient. Rehab. Med. 2003, 13, 87-101.

28. Yu, S.M.; Lee, J.Y.; Kwon, K.R.; Lee, H.S. Comparative study on acupuncture, bee venom acupuncture, and bee venom pharmacopuncture on the treatment of herniation of nucleus pulpous. J. Korean Acupunct. Moxibustion Soc. 2006, 23, 39-54.

29. Kwon, Y.B.; Kim, J.H.; Yoon, J.H.; Lee, J.D.; Han, H.J.; Mar, W.C.; Beitz, A.J.; Lee, J.H. The analgesic efficacy of bee venom acupuncture for knee osteoarthritis: A comparative study with needle acupuncture. Am. J. Chin. Med. 2001, 29, 187-199. [CrossRef]

30. Conrad, V.J.; Hazan, L.L.; Latorre, A.J.; Jakubowska, A.; Kim, C.M.H. Efficacy and safety of honey bee venom (Apis mellifera) dermal injections to treat osteoarthritis knee pain and physical disability: A randomized controlled trial. J. Altern. Complement. Med. 2019, 25, 845-855. [CrossRef]

31. Wesselius, T.; Heersema, D.J.; Mostert, J.P.; Heerings, M.; Admiraal-Behloul, F.; Talebian, A.; van Buchem, M.A.; De Keyser, J. A randomized crossover study of bee sting therapy for multiple sclerosis. Neurology 2005, 65, 1764-1768. [CrossRef]

32. Kim, K.T.; Song, H.S. A randomized controlled double blinding study of bee venom acupuncture therapy on sprain of c-spine. $J$. Korean Acupunct. Moxibustion Soc. 2005, 22, 189-195.

33. Mohamed, E.A.; Ewida, M.M. Efficacy of bee venom phonphoresis in treatment of chronic pelvic inflammatory diseases. Int. J. Pharm. Tech. Res. 2016, 9, 66-71.

34. Nitecka-Buchta, A.; Buchta, P.; Tabeńska-Bosakowska, E.; Walczyńska-Dragoń, K.; Baron, S. Myorelaxant effect of bee venom topical skin application in patients with RDC/TMD Ia and RDC/TMD Ib: A randomized, double blinded study. Biomed. Res. Int. 2014, 2014, 296053. [CrossRef]

35. An, B.J.; Song, H.S. Effect of bee venom acupuncture therapy on patients with sprain of the wrist. J. Pharmacopunct. 2006, 9, 167-171.

36. Yasin, M.M.; Elhosary, E.A.; Hamada, H.A.; Yousef, A.M.; Shahin, M.; Mosaad, D. Effect of bee venom phonophoresis in obese polycystic ovarian women: A single blind randomized controlled trial. J. Appl. Pharm. Sci. 2018, 8, 159-164.

37. Song, H.S. The effect of bee venom acupuncture (BVA) on acute ankle sprain: A randomized controlled trial and double blinding-pilot study. J. Pharmacopunct. 2005, 8, 11-16.

38. Seo, J.W.; Park, M.J.; Sung, I.H.; Kim, N.O.; Ahn, C.K. A clinical study of bee venom acupunture therapy on the treatment of acute ankle sprain. J. Korean Acupunct. Moxibustion Soc. 2006, 23, 95-102. 
39. Cho, S.Y.; Shim, S.R.; Rhee, H.Y.; Park, H.J.; Jung, W.S.; Moon, S.K.; Park, J.M.; Ko, C.N.; Cho, K.H.; Park, S.U. Effectiveness of acupuncture and bee venom acupuncture in idiopathic Parkinson's disease. Parkinsonism Relat. Disord. 2012, 18, 948-952. [CrossRef] [PubMed]

40. Cho, S.Y.; Lee, Y.E.; Doo, K.H.; Lee, J.H.; Jung, W.S.; Moon, S.K.; Park, J.M.; Ko, C.N.; Kim, H.; Rhee, H.Y.; et al. Efficacy of combined treatment with acupuncture and bee venom acupuncture as an adjunctive treatment for parkinson's disease. J. Altern. Complement. Med. 2018, 24, 25-32. [CrossRef] [PubMed]

41. Hartmann, A.; Mullner, J.; Meier, N.; Hesekamp, H.; van Meerbeeck, P.; Habert, M.O.; Kas, A.; Tanguy, M.L.; Mazmanian, M.; Oya, H.; et al. Bee venom for the treatment of parkinson disease-a randomized controlled clinical trial. PLoS ONE 2016, 11, e0158235.

42. Park, J.A.; Lee, C.H.; Kwon, G.S.; Lee, K.A.; Jang, K.J. The effects of sweet bee venom pharmacopuncture on post-stroke hemiplegic shoulder pain. J. Korean Acupunct. Moxibustion Soc. 2011, 2, 37-47.

43. Ko, C.N.; Min, I.K.; Park, S.W.; Jung, W.S.; Moon, S.K.; Park, J.M.; Cho, K.H.; Kim, Y.S.; Bae, H.S. Effectiveness of bee venom acupuncture on shoulder pain after stroke. J. Korean Orient. Med. 2007, 28, 11-24.

44. Eom, J.Y.; Won, S.H.; Kwon, K.R.; Lee, H.S. Comparative study on acupuncture, bee venom acupuncture, and bee venom herbal acupuncture in the treatment of post-stroke hemiplegic shoulder pain. J. Pharmacopunct. 2006, 9, 139-154.

45. Lee, D.Y.; Lee, G.K.; Yeom, S.C.; Kim, D.H.; Kim, D.J. A clinical study of bee venom acupuncture therapy on shoulder pain patients in stroke sequelae. J. Korean Acupunct. Moxibustion Soc. 2006, 23, 69-80.

46. Cho, S.W.; Go, K.H.; Nam, J.H.; Kim, M.S.; Lee, S.Y.; Lee, I.S. The effectiveness of Zingiberis Rhizoma herbal acupuncture therapy and bee venom herbal acupuncture therapy on the poststroke hemiplegic shoulder pain. J. Orient. Rehab. Med. 2005, 15, 77-87.

47. Lee, S.H.; Hong, S.J.; Kim, S.Y.; Yang, H.I.; Lee, J.D.; Choi, D.Y.; Lee, D.I.; Lee, Y.H. Randomized controlled double blind study of bee venom therapy on rheumatoid arthritis. J. Korean Acupunct. Moxibustion Soc. 2003, 20, 80-88.

48. Chen, S.Y.; Zhou, P.; Qin, Y. Treatment of rheumatoid arthritis by bee-venom acupuncture. Zhen Ci Yan Jiu 2018, 43, 251-254 [PubMed]

49. Liu, M.; Counsell, C.; Wardlaw, J.; Sandercock, P. A systematic review of randomized evidence for fibrinogen-depleting agents in acute ischemic stroke. J. Stroke Cerebrovasc. Dis. 1998, 7, 63-69. [CrossRef]

50. Shetty, V.; Sriram, S.G. Effectiveness of intravenous haemocoagulase on haemorrhage control in bi-maxillary orthognathic surgery-a prospective, randomised, controlled, double-blind study. J. Craniomaxillofac. Surg. 2015, 43, 2000-2003. [CrossRef]

51. Qiu, M.; Zhang, X.; Cai, H.; Xu, Z.; Lin, H. The impact of hemocoagulase for improvement of coagulation and reduction of bleeding in fracture-related hip hemiarthroplasty geriatric patients: A prospective, single-blinded, randomized, controlled study. Injury 2017, 48, 914-919. [CrossRef]

52. Zhang, S.Y.; Jin, L.; Yan, X.W. The evaluation of thrombolytic effect of snake venom antithrombus enzyme in treatment of acute myocardial infarction. Zhonghua Nei Ke Za Zhi 1994, 33, 244-247.

53. Huang, D.X.; Gai, L.Y.; Wang, S.R.; Li, T.D.; Yang, T.S.; Zhi, G.; Du, L.S.; Li, L.H. Defibrase, a purified fibrinolytic protease from snake venom in acute myocardial infarction. Acta Cardiol. 1992, 47, 445-458.

54. Gamba, G.; Fornasari, P.M.; Grignani, G.; Dolci, D.; Colloi, D. Haemostasis during transvesical prostatic adenomectomy. A controlled trial on the effect of drugs with antifibrinolytic and thrombin-like activities. Blut 1979, 39, 89-98. [CrossRef]

55. Kim, J.S.; Yoon, S.S.; Kwon, S.U.; Ha, J.H.; Suh, E.J.; Chi, H.S. Treatment of acute cerebral infarction with arginine esterase: A controlled study with heparin. Cerebrovasc. Dis. 2001, 11, 251-256. [CrossRef]

56. Xu, J.; Qian, S.S.; Chen, Y.G.; Li, D.Y.; Yan, Q. Systematic review and meta-analysis of efficacy and safety of Huachansu in treating cancer-related pain. Zhongguo Zhong Yao Za Zhi 2019, 44, 2627-2636. [PubMed]

57. Wu, J.; Zhang, D.; Ni, M.; Xue, J.; Wang, K.; Duan, X.; Liu, S. Effectiveness of Huachansu injection combined with chemotherapy for treatment of gastric cancer in China: A systematic review and meta-analysis. J. Tradit. Chin. Med. 2020, 40, 749-757. [PubMed]

58. Zhang, D.; Wang, K.; Zheng, J.; Wu, J.; Duan, X.; Ni, M.; Liu, S.; Zhang, B.; Zhao, Y. Comparative efficacy and safety of Chinese herbal injections combined with transcatheter hepatic arterial chemoembolization in treatment of liver cancer: A bayesian network meta-analysis. J. Tradit. Chin. Med. 2020, 40, 167-187. [PubMed]

59. Wang, T.; Zhang, L.; Han, L.; Liu, X.; Zhang, H.; Zhang, J.; Yu, H. Clinical effect of intravenous infusion of zoledronic acid combined with oral medication of cinobufagin in the treatment of metastatic bone tumors. Pak. J. Pharm. Sci. 2018, 31, 1609-1612. [PubMed]

60. Chen, Z.; Zhai, X.F.; Su, Y.H.; Wan, X.Y.; Li, J.; Xie, J.M.; Gao, B. Clinical observation of cinobufacini injection used to treat moderate and advanced primary liver cancer. Zhong Xi Yi Jie He Xue Bao 2003, 1, 184-186. [CrossRef] [PubMed]

61. Meng, Z.; Garrett, C.R.; Shen, Y.; Liu, L.; Yang, P.; Huo, Y.; Zhao, Q.; Spelman, A.R.; Ng, C.S.; Chang, D.Z.; et al. Prospective randomised evaluation of traditional Chinese medicine combined with chemotherapy: A randomised phase II study of wild toad extract plus gemcitabine in patients with advanced pancreatic adenocarcinomas. Br. J. Cancer 2012, 107, 411-416. [CrossRef]

62. Vetter, R.S.; Visscher, P.K.; Camazine, S. Mass envenomations by honey bees and wasps. West. J. Med. 1999, 170, $223-227$.

63. Gale, A.N. Insect-sting encephalopathy. Br. Med. J. Clin. Res. Ed. 1982, 284, 20-21. [CrossRef]

64. Reisman, R.E. Unusual reactions to insect stings. Curr. Opin. Allergy Clin. Immunol. 2005, 5, 355-358. [CrossRef]

65. Lee, G.; Bae, H. Bee Venom Phospholipase A2: Yesterday's Enemy Becomes Today's Friend. Toxins 2016, 8, 48. [CrossRef]

66. Lee, K.G.; Cho, H.J.; Bae, Y.S.; Park, K.K.; Choe, J.Y.; Chung, I.K.; Kim, M.; Yeo, J.H.; Park, K.H.; Lee, Y.S.; et al. Bee venom suppresses LPS-mediated NO/iNOS induction through inhibition of PKC- $\alpha$ expression. J. Ethnopharmacol. 2009, 123, 15-21. [CrossRef] [PubMed] 
67. Lim, B.S.; Moon, H.J.; Li, D.X.; Gil, M.; Min, J.K.; Lee, G.; Bae, H.; Kim, S.K.; Min, B.I. Effect of bee venom acupuncture on oxaliplatin-induced cold allodynia in rats. Evid. Based Complement. Alternat. Med. 2013, 2013, 369324. [CrossRef] [PubMed]

68. Huh, J.E.; Baek, Y.H.; Lee, M.H.; Choi, D.Y.; Park, D.S.; Lee, J.D. Bee venom inhibits tumor angiogenesis and metastasis by inhibiting tyrosine phosphorylation of VEGFR-2 in LLC-tumor-bearing mice. Cancer Lett. 2010, 292, 98-110. [CrossRef] [PubMed]

69. Alqutub, A.N.; Masoodi, I.; Alsayari, K.; Alomair, A. Bee sting therapy-induced hepatotoxicity: A case report. World J. Hepatol. 2011, 3, 268-270. [CrossRef]

70. Bae, E.J.; Son, S.B.; Seo, S.H.; Son, S.W.; Kim, I.H. A case of foreign body granuloma with skin necrosis occurring after bee sting therapy. Korean J. Dermatol. 2009, 47, 350-353.

71. Cheng, Y.M.; Ren, X.H. Arrhythmia by bee sting acupuncture. J. Clin. Acupunct. Moxibustion 2004, $20,54$.

72. Zhang, J.W.; Shi, D.Y.; Wang, L.Y.; Liu, R.C.; Zhang, L. Investigation of anaphylaxis by bee sting acupuncture in 9 case. Shanghai J. Acupunct. Moxibustion 1995, 3, 126.

73. Jung, J.W.; Jeon, E.J.; Kim, J.W.; Choi, J.C.; Shin, J.W.; Kim, J.Y.; Park, I.W.; Choi, B.W. A fatal case of intravascular coagulation after bee sting acupuncture. Allergy Asthma. Immunol. Res. 2012, 4, 107-109. [CrossRef]

74. Mehta, S.R.; Sashindran, V.K. Clinical features and management of snake bite. Med. J. Armed. Forces India 2002, 58, 247-249. [CrossRef]

75. Amin, M.R.; Mamun, S.M.H.; Rashid, R.; Rahman, M.; Ghose, A.; Sharmin, S.; Rahman, M.R.; Faiz, M.A. Anti-snake venom: Use and adverse reaction in a snake bite study clinic in Bangladesh. J. Venom. Anim. Toxins Incl. Trop. Dis. 2008, 14, 660-672. [CrossRef]

76. Camargo, A.C.M.; Ianzer, D.; Guerreiro, J.R.; Serrano, S.M.T. Bradykinin-potentiating peptides: Beyond captopril. Toxicon 2012, 59, 516-523. [CrossRef] [PubMed]

77. Castro, H.C.; Zingali, R.B.; Albuquerque, M.G.; Pujol-Luz, M.; Rodrigues, C.R. Snake venom thrombin-like enzymes: From reptilase to now. Cell Mol. Life Sci. 2004, 61, 843-856. [CrossRef] [PubMed]

78. Chan, Y.S.; Cheung, R.C.F.; Xia, L.; Wong, J.H.; Ng, T.B.; Chan, W.Y. Snake venom toxins: Toxicity and medicinal applications. Appl. Microbiol. Biotechnol. 2016, 100, 6165-6181. [CrossRef] [PubMed]

79. FDA-Approved Drugs. Available online: https://www.accessdata.fda.gov/scripts/cder/daf/index.cfm (accessed on 11 November 2020).

80. Gowda, R.M.; Cohen, R.A.; Khan, I.A. Toad venom poisoning: Resemblance to digoxin toxicity and therapeutic implications. Heart 2003, 89, e14. [CrossRef] [PubMed]

81. Zheng, Y.; Deng, L.; Cao, H.; Xu, N.; Zhang, D.; Tian, H.; Li, B.; Lu, Z.; Ye, W.; Yu, L.; et al. Screening of bufadienolides from toad venom identifies gammabufotalin as a potential anti-inflammatory Agent. Planta Med. 2020. [CrossRef]

82. Banfi, F.F.; Guedes Kde, S.; Andrighetti, C.R.; Aguiar, A.C.; Debiasi, B.W.; Noronha Jda, C.; Rodrigues Dde, J.; Vieira, G.M., Jr.; Sanchez, B.A. Antiplasmodial and cytotoxic activities of toad venoms from southern amazon, Brazil. Korean J. Parasitol. 2016, 54, 415-421. [CrossRef]

83. Schmeda-Hirschmann, G.; Quispe, C.; Theoduloz, C.; de Sousa, P.T., Jr.; Parizotto, C. Antiproliferative activity and new argininyl bufadienolide esters from the "cururú" toad Rhinella (Bufo) schneideri. J. Ethnopharmacol. 2014, 155, 1076-1085. [CrossRef]

84. Boussios, S.; Pentheroudakis, G.; Katsanos, K.; Pavlidis, N. Systemic treatment-induced gastrointestinal toxicity: Incidence, clinical presentation and management. Ann. Gastroenterol. 2012, 25, 106-118.

85. Warpinski, J.R.; Bush, R.K. Stinging insect allergy. J. Wilderness Med. 1990, 1, 249-257. [CrossRef]

86. Chippaux, J.P. Epidemiology of envenomations by terrestrial venomous animals in Brazil based on case reporting: From obvious facts to contingencies. J. Venom. Anim. Toxins Incl. Trop. Dis. 2015, 21, 1-17. [CrossRef]

87. Hong, K.E. Analysis study of survey for safety and efficacy of pharmacopuncture. Aust. J. Pharm. 2010, 13, 91-102. [CrossRef]

88. Ahn, U.C.; Kin, H.D.; Kim, J.H.; Rho, T.W.; Han, S.Y.; Kim, Y.K. A survey on the management status of extramural herbal dispensaries. Herb. Formula Sci. 2016, 24, 1-16. [CrossRef]

89. Sung, S.H.; Han, J.E.; Ryu, J.Y.; Sung, A.D.M.; Park, J.Y.; Ha, I.H.; Kim, K.H.; Park, J.K.; Shin, B.C. Current status and future perspective of external herbal dispensaries preparing traditional herbal medicine in South Korea: The first national-wide survey results. BMC Complement. Med. Ther. 2020, 20, 354. [CrossRef] [PubMed] 